\title{
The modulation of electromagnetic ion cyclotron waves by Pc 5 ULF waves
}

\author{
T. M. Loto'aniu ${ }^{1,2}$, B. J. Fraser ${ }^{3}$, and C. L. Waters ${ }^{3}$ \\ ${ }^{1}$ Cooperative Institute for Research in Environmental Sciences, University of Colorado, Boulder, CO, USA \\ ${ }^{2}$ Space Weather Prediction Center, National Oceanic and Atmospheric Administration, Boulder, CO, USA \\ ${ }^{3}$ Center for Space Physics, School of Mathematical and Physical Sciences, University of Newcastle, NSW, Australia
}

Received: 4 November 2007 - Revised: 19 November 2008 - Accepted: 26 November 2008 - Published: 7 January 2009

\begin{abstract}
The modulation of electromagnetic ion cyclotron (EMIC) waves by longer-period ULF waves has been proposed as a method for producing pearl structured Pc 1-2 EMIC waves. This study examines frequency and phase relationship between Pc 1 EMIC wavepacket envelopes and simultaneously occurring Pc 5 ULF waves using magnetic data measured by the CRRES spacecraft. Intervals from three days in 1991 where CRRES observed pearls are presented along with simple statistics for 58 EMIC wavepackets. The observations were dominated by EMIC waves propagating away from the equatorial region. Comparisons between pearl wavepacket envelopes and Pc 5 waves show excellent agreement. The pearl wavepacket duration times, $\tau_{\text {dur }}$, were statistically correlated with Pc 5 wave periods, $T_{\mathrm{Pc} 5}$, resulting in a correlation coefficient of $R=0.7$ and best fit equation $\tau_{\text {dur }}=0.8 \cdot T_{\mathrm{Pc} 5}+6 \mathrm{~s}$. In general, phase differences varied although time intervals of constant in-phase or anti-phase correlation were observed. Anti-phase modulation may be explained by a decreasing background magnetic field due to the negative cycle of the ULF wave decreasing Alfvén velocity and minimum resonant energy. In-phase modulation could be the result of adiabatic modulation of temperature anisotropy in-phase with variations in the background field. Non-adiabatic processes may contribute to intervals that showed varying phase differences with time. Results suggest that future theoretical developments should take into account the full range of possible wave particle interactions inside the magnetosphere.
\end{abstract}

Keywords. Magnetospheric physics (MHD waves and instabilities; Plasma waves and instabilities) - Space plasma physics (Wave-wave interactions)

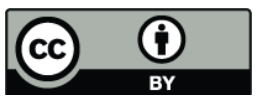

Correspondence to: T. M. Loto'aniu (paul.lotoaniu@noaa.gov)

\section{Introduction}

Electromagnetic ion cyclotron (EMIC) waves generated in the equatorial region of Earth's magnetosphere as left-hand (LH) polarised waves propagate field-line guided towards the ionosphere (e.g. Mauk and McPherron, 1980). When observed on the ground, EMIC waves are usually measured with frequencies in the Pc $1-2$ or $0.1-5.0 \mathrm{~Hz}$ range (Jacobs et al., 1964). The Pc 1-2 pulsations can be categorised into two types: structured and unstructured (Saito, 1969). The structured Pc 1-2 waves are frequency band limited, can last from tens of minutes to several hours at mid to high latitudes (Troitskaya, 1961) and are sometimes referred to as "pearl pulsations" as time series magnetograms often show repeating periodic structure that resembles a necklace pearl pattern (Tepley and Landshoff, 1966).

Conjugate studies from the 1960s observed the pearl wavepackets to alternate between Northern and Southern Hemispheres (e.g. Tepley, 1964). This alternating pearl type structure between hemispheres, was interpreted by Jacobs and Watanabe (1964) and Obayashi (1965) by the "Bouncing Wave Packet" (BWP) model. According to the BWP model, wavepackets bounce back and forth along a geomagnetic field-line between ionospheric mirror points in a manner similar to VLF whistlers. Only a small part of the EMIC wave energy reflects from the ionosphere and when this signal reaches the source region it is re-amplified and continues traveling to the opposite ionosphere. The remainder of the energy is transmitted into the ground (e.g. McPherron et al., 1972; Bossen et al., 1976).

Recent observations do not support the BWP model and have all but discredited the concept of bouncing wavepackets to explain pearl formation (e.g., see Mursula, 2007). EMIC wave Poynting vector measurements show wavepacket propagation predominately unidirectionally away from the equatorial region (Fraser et al., 1996; Mursula et al., 1997, 2001). More recently Loto'aniu et al. (2005) conducted a statistical

Published by Copernicus Publications on behalf of the European Geosciences Union. 
study of 248 EMIC wave events observed by the CRRES satellite. They estimated the Poynting vector in the dynamic spectral domain permitting studies of energy propagation of simultaneous waves located in different frequency bands. The direction of wave energy propagation was found to be independent of wave frequency but dependent on magnetic latitude. EMIC wave energy propagates bidirectional both away and towards the equator for events observed below $11^{\circ} \mid$ MLat $\mid$, but unidirectionally away from the equator for all events located at latitudes greater than $11^{\circ}$ |MLat|. They found no measurable wave energy propagating equatorwards beyond the source region, in contradiction to the BWP model.

A number of different alternatives to the BWP model have been proposed. One of these alternatives suggest EMIC pearls are generated through modulation of EMIC wave growth rate by longer-period ULF waves (e.g. Mursula et al., 1997). ULF wave observations in the magnetosphere often show simultaneous presence of both Pc 1-2 waves and longer period pulsations $(<0.1 \mathrm{~Hz})$ (e.g. Barfield and McPherron, 1972; Maltzeva et al., 1982; Fraser et al., 1986, 1992).

Barfield and McPherron (1972) using ATS 1 spacecraft data found that EMIC waves appeared during 55\% of Pc 5 $(2-10 \mathrm{mHz})$ wave events while Maltzeva et al. (1982) found that observations of Pc 4 pulsations during the recovery phase of geomagnetic storms were often accompanied by EMIC waves. In studying EMIC wave bursts observed by the Viking spacecraft and on the ground Mursula et al. (1997) suggested that long-period ULF wave modulation could best explain the conjugate observations of repetition periods. Mursula et al. (2001) studied an EMIC event using conjugate ground-space observations and found that longperiod ULF wave modulation with possibly additional maser effects could explain their observations. The magnetospheric maser (Polyakov et al., 1983), where EMIC waves form a resonator between the two ionospheres may provide positive feedback to the equatorial generation region enhancing the EMIC waves (Mursula et al., 2001).

In this study, pearl Pc 1 EMIC waves measured onboard CRRES on three different days are presented, which represent typical pearl events observed by the CRRES spacecraft. Five time intervals, which showed constant in-phase or antiphase correlation between pearl wavepacket envelope periods and Pc 5 wave periods are further analysed. For the first time direct spectral frequency comparisons between pearl wavepacket envelopes and Pc 5 waves are presented. The statistical correlation between 58 pearl EMIC wavepackets and Pc 5 wave periods is also examined. Results show clear correlation between pearl Pc 1 EMIC wavepacket envelopes and simultaneously observed compressional Pc 5 ULF waves. Discussion focuses on exploring whether Pc 5 wave modulation of parameters important to EMIC wave growth may generate the pearl structure. Heavy ion effects on the modulation process are also discussed.

\section{Data analysis and results}

The Combined Release and Radiation Effects Satellite (CRRES) was operational for about 14 months during 1990 to 1991 and traversed the middle magnetosphere over $L=3-8$ and up to $\sim 30^{\circ}$ |MLat| (Singer et al., 1992; Wygant et al., 1992; Anderson et al., 1992). Three component vector magnetic field measurements onboard CRRES were provided by the Air Force Geophysics Laboratory (AFGL) fluxgate magnetometer sampled to provide data with $8 \mathrm{~Hz}$ Nyquist frequency (see, Singer et al., 1992). The University of Iowa/AFGL Plasma Waves Experiment (PWE) was used to estimate cold electron densities at the CRRES locations through observations of the upper hybrid resonance frequency $\left(f_{\mathrm{UHR}}\right)$ (see Anderson et al., 1992). In the middle magnetosphere, $f_{\mathrm{UHR}}$ is approximately equal to the plasma frequency, $f_{p e}$, and electron density can be approximated as $N_{e}=f_{p e}^{2} / 81$ (Gurnett and Inan, 1988). The magnetic field data are presented in field-aligned coordinates, where the $\mathrm{z}-$ component is aligned parallel to the ambient magnetic field vector.

The EMIC wave Poynting vectors were calculated in the spectral domain as discussed by Loto' aniu et al. (2005). The EMIC wavepacket envelopes were estimated as the magnitude of the EMIC wave analytic signals. Pc 5 ULF wave data were obtained by detrending the magnetic field data and lowpass filtering with a $30 \mathrm{mHz}$ cutoff.

For individual event comparisons, spectral analysis was used to idenfity both Pc 5 wave and Pc 1 envelope frequencies. Once frequencies were identified, the data was further band-pass filtered to remove background noise and emphasise the frequency region of interest. Power spectral widths were defined as $\pm 0.5 \times$ FWHM (Full width half maximum), while the FWHMs were determined by fitting power spectra to 6th order Gaussians.

For statistical analysis, the Pc 5 periods were estimated by measuring times between consecutive Pc 5 cycle peaks during each EMIC wavepacket time interval. EMIC wavepacket identification and duration (in time) follows that of Loto' aniu et al. (2005), where observed wave power must be above $0.06 \mathrm{nT}^{2} / \mathrm{Hz}$ for at least $60 \mathrm{~s}$. Wavepacket repetition is defined as the observed time between wavepacket midpoints.

\subsection{EMIC wave interval with $f<f_{\mathrm{He}+}$}

On 12 August 1991, orbit 926, CRRES observed EMIC waves with dominant power in the $\mathrm{He}^{+}$wave band or with wave frequency $f<f_{\mathrm{He}+}$, where $f_{\mathrm{He}+}$ is the $\mathrm{He}^{+}$ion cyclotron frequency. The waves occurred over 11:30-12:05 UT (13:30-14:04 MLT) when CRRES was outbound and near apogee covering $L=5.40-6.08$ in the Southern Hemisphere with MLat $\sim-20^{\circ}$, shown in Fig. 1 . According to the PWE spectrogram, shown in Fig. 2, when the EMIC waves were observed CRRES was within the plasmatrough but inside an enhanced plasma region. The enhanced plasma density is 


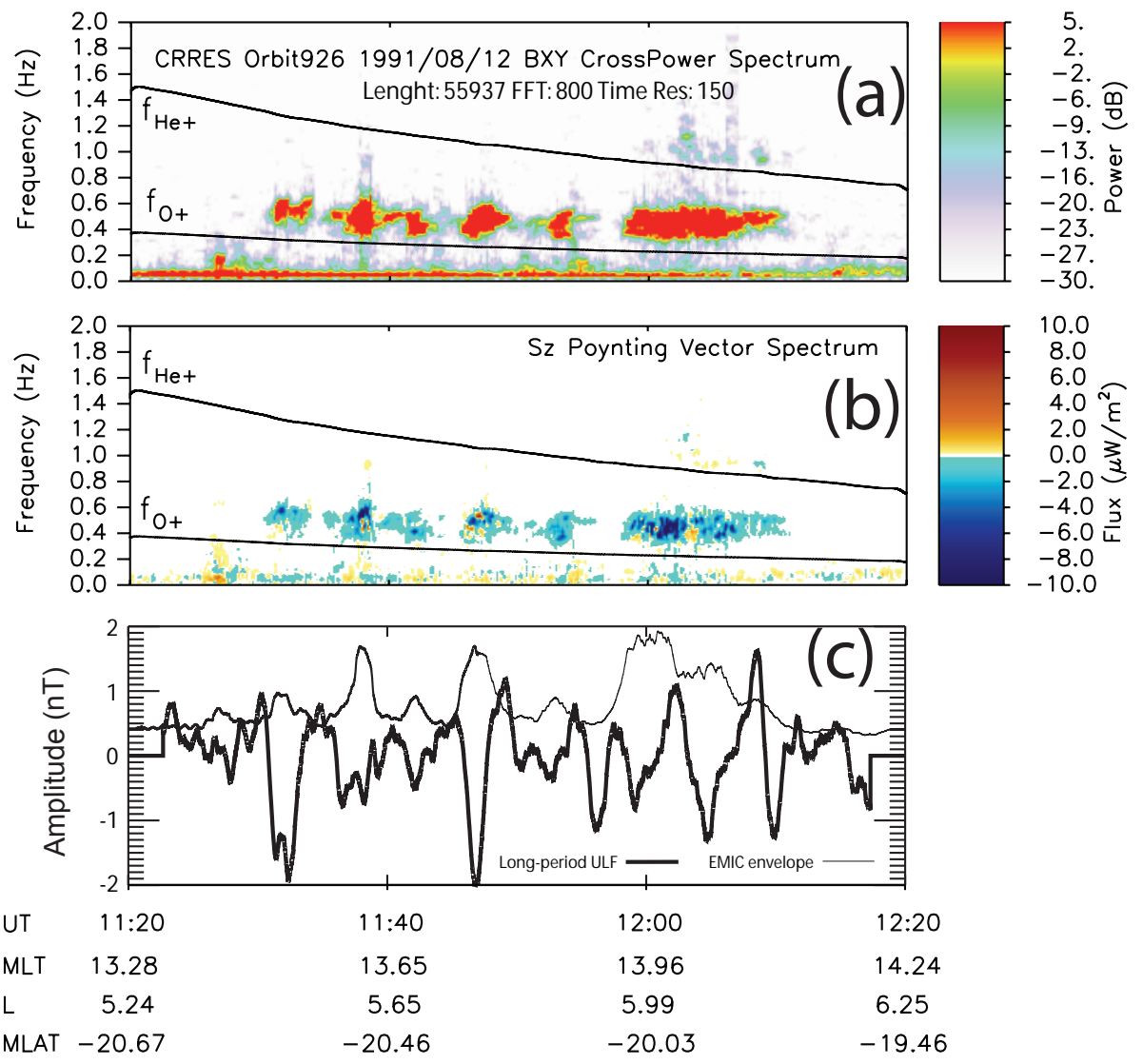

Fig. 1. Orbit 926, 12 August 1991 event. (a) transverse magnetic cross power, (b) field-aligned Poynting vector $S_{z}$ spectrogram and (c) the ULF magnetic compressional wave (thickline) and the EMIC wavepacket transverse magnetic amplitude envelope (thinline). The black lines in (a) and (b) represent the local $\mathrm{He}^{+}\left(f_{\mathrm{He}+}\right)$ and $\mathrm{O}^{+}\left(f_{\mathrm{O}+}\right)$ cyclotron frequencies.

inferred by the observed increase in the upper hybrid frequency over the time interval 11:30-12:50 UT in Fig. 2. A sudden storm commencement (SSC) was seen the previous day starting at 02:50 UT. The EMIC waves consisted of at least six wave packets shown in the crosspower spectrogram, Fig. 1a. Wave frequencies centered on $f=0.45-$ $0.50 \mathrm{~Hz}$ or normalised (by the $\mathrm{H}^{+}$cyclotron frequency) local and equatorial frequencies $X_{\text {local }}=0.10-0.13$ and $X_{e q}=0.17-$ 0.21 , respectively. The transverse components dominated with field amplitudes ranging from 1.0-3.3 nT for $\delta B_{x}$ and $\delta B_{y}$. There was minimal power in the field-aligned direction (not shown).

The field-aligned Ponyting vector $S_{z}$ is shown in Fig. 1b with the colour bar indicating magnitude and direction of energy flux. Since CRRES was located south of the magnetic equator, yellow to red is positive (south to north) energy flux and light blue to dark-blue negative (north to south) energy flux. Clearly, energy propagates predominately away from the equatorial region towards the Southern Hemisphere, which conflicts with the BWP model. Figure 1c shows the transverse EMIC wave envelope (thinline) and simultane- ously observed Pc 5 ULF wave compressional component (thickline). Peaks in the EMIC wavepacket envelope aligned with troughs of the Pc 5 compressional wave cycle suggesting anti-phase modulation of the EMIC wave by the Pc 5 wave. Since the Pc 5 compressional wave modulates the background field, this anti-phase modulation suggests that the EMIC wavepacket amplitudes were maximised during minimum background field.

Figure 3 shows normalised static frequency power spectra for the observed Pc 5 wave (thickline) and Pc 1 wavepacket envelope (thinline) on 12 August. The Pc 5 wave frequency of $3.8 \pm 0.4 \mathrm{mHz}(263 \pm 25 \mathrm{~s})$ is close to the Pc 1 envelope repeat time of $313 \pm 50 \mathrm{~s}(3.2 \pm 0.6) \mathrm{mHz}$, while the general frequency coverage shows excellent agreement. Hence, Pc 1 pearls observed by CRRES on 12 August have wavepacket repeat times and/or duration times approximately equal to the periodicity of a simultaneously observed Pc 5 wave. The implications of this result along with observed anti-phase correlation to pearl generation are discussed in Sect. 3 . 


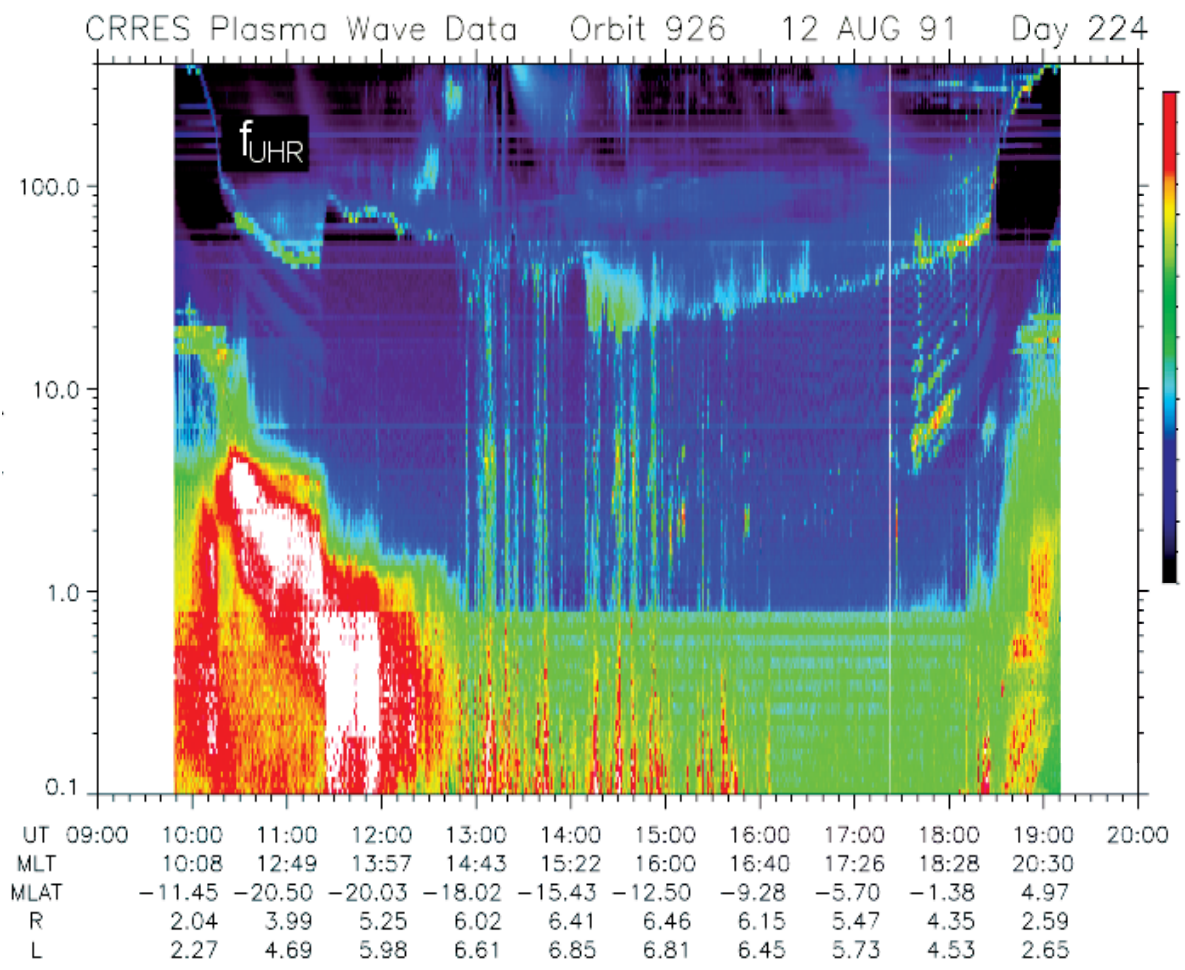

Fig. 2. Orbit 926, 12 August 1991 plasma waves spectrogram. The $f_{\mathrm{UHR}}$ is indicated, which estimates the plasmapause location.

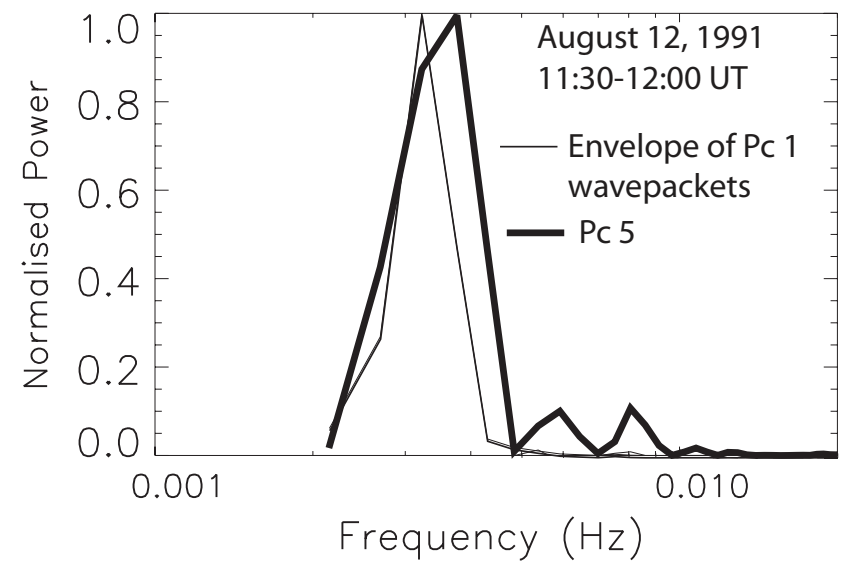

Fig. 3. Orbit 926, 12 August 1991 normalised power spectra over the interval 11:30-12:00 UT. The thinline is the Pc 1 transverse wave amplitude envelope spectrum while the thickline is the Pc 5 wave spectrum.

\subsection{EMIC wave intervals with $f>f_{\mathrm{He}+}$}

Pearl Pc 1 EMIC waves were observed by CRRES on $11 \mathrm{Au}-$ gust (orbit 923) and 1 October (orbit 1043) 1991 (see Fig. 4). In both cases, dominate wavepacket power appeared at frequencies with $f>f_{\mathrm{He}+}$. On 11 August CRRES observed
EMIC waves between 04:50-06:07 UT (14.4-15.3 MLT), or in the local afternoon with $\mid$ MLat $\mid \sim 26^{\circ}$ and $L=6.3-7.3$. CRRES observed EMIC waves on 1 October close to the equator $\left(\mid\right.$ MLat $\left.\mid \sim 3^{\circ}\right)$ and in the local afternoon (18:10-19:40 UT) sector. The EMIC wave intervals were observed shortly after or during SSCs, while the PWE spectrograms (not shown) indicate CRRES was inside the plasmatrough on both occasions.

The Poynting vector spectrum for data recorded on 11 August (Fig. 4b) was dominated by unidirectional propagation away from the equator. On 1 October, EMIC wave Poynting vector (Fig. 4e) were again dominated by propagation away from the equator, although in this case significant propagation towards the equatorial region was also observed. The observed bidirectional energy flux close to the equator is consistent with results of Loto'aniu et al. (2005). The near-equatorial region generates EMIC waves that propagate in different directions. However, once outside this region wave energy propagation is predominately unidirectional away from the equatorial region.

Simultaneous Pc 5 waves were also measured during the pearl intervals on 11 August and 1 October (see Figs. 4c and $4 \mathrm{f}$ ). However, in these cases, phase differences between Pc 5 wave cycles and Pc 1 wavepacket envelopes were not constant throughout the observed time intervals. In order to simplify discussion of phasing between Pc 5 waves and pearls, example time intervals were chosen for closer study. 

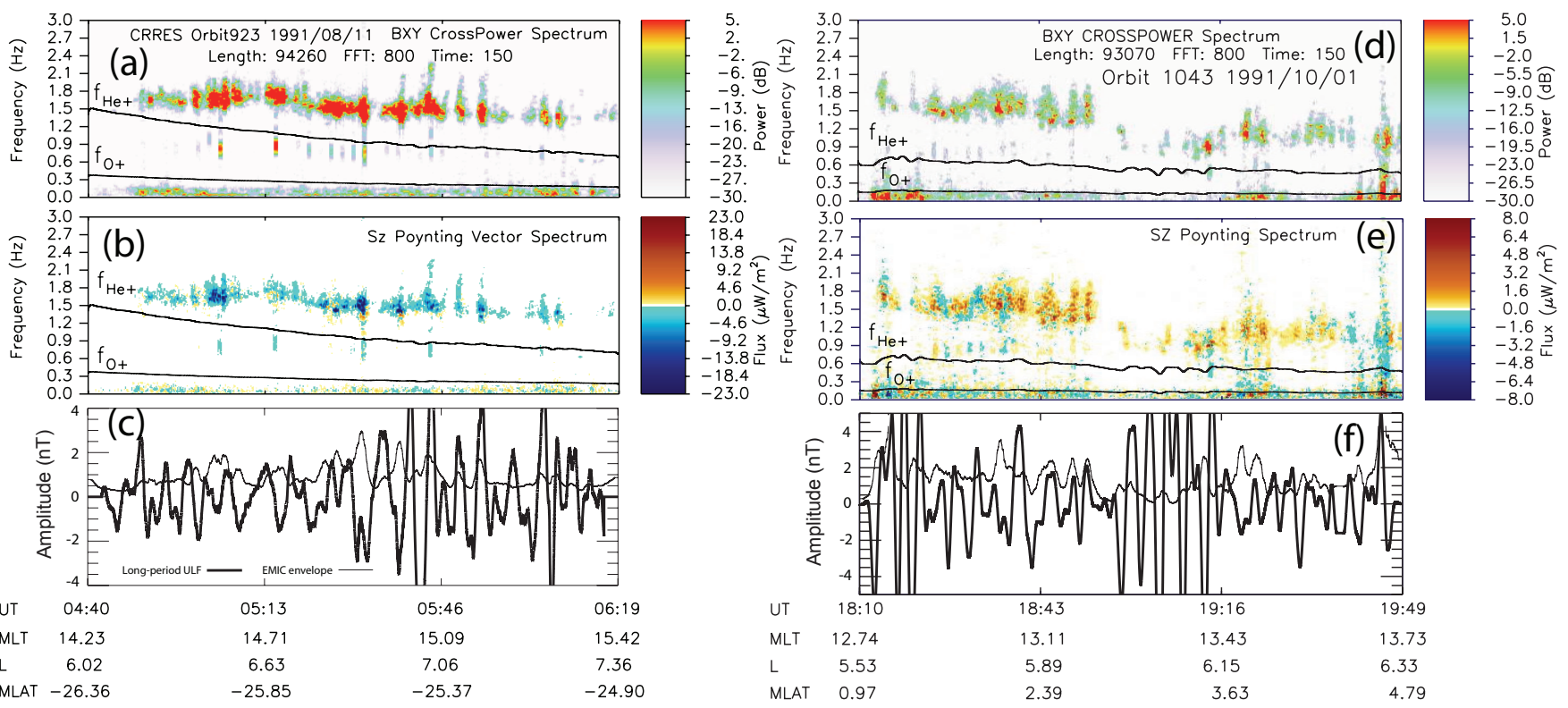

Fig. 4. EMIC waves observed by CRRES during 11 August and 1 October 1991. (a) and (d) transverse magnetic cross powers, (b) and (e) field-aligned Poynting vector spectrograms and (c) and (f) ULF magnetic compressional waves (thickline) and the EMIC wavepacket transverse magnetic envelopes (thinline). The black lines in (a), (b), (d) and (e) represent the local $\mathrm{He}^{+}\left(f_{\mathrm{He}^{+}}\right)$and $\mathrm{O}^{+}\left(f_{\mathrm{O}+}\right)$ cyclotron frequencies.

Table 1. Example intervals of simultaneous pearl Pc 1 EMIC wave envelopes and Pc 5 wave observations.

\begin{tabular}{|c|c|c|c|c|c|c|c|c|c|}
\hline Event & Orbit & Date & UT & MLT & $\mathrm{L}$ & $\operatorname{MLAT}\left({ }^{\circ}\right)$ & $\begin{array}{c}\text { Pc } 1 \text { envelope } \\
\text { frequency }(\mathrm{mHz})^{\mathrm{a}}\end{array}$ & $\begin{array}{c}\text { Pc } 5 \text { wave } \\
\text { frequency }(\mathrm{mHz})^{\mathrm{a}}\end{array}$ & Phase \\
\hline 1 & 926 & 12 August & 11:30-12:00 & 14 & 5.8 & -20 & $3.2 \pm 0.6$ & $3.8 \pm 0.4$ & anti-phase \\
\hline 2 & 923 & 11 August & $05: 00-05: 40$ & 15 & 6.7 & -25 & $2.0 \pm 0.7$ & $2.0 \pm 0.9$ & anti-phase \\
\hline 3 & 923 & 11 August & $05: 40-06: 00$ & 15 & 7.1 & -25 & $3.8 \pm 0.7$ & $3.8 \pm 0.8$ & in-phase \\
\hline 4 & 1043 & 1 October & $18: 40-18: 55$ & 13 & 6.0 & 3 & $4.3 \pm 1.4$ & $3.3 \pm 0.8$ & in-phase \\
\hline 5 & 1043 & 1 October & $19: 15-19: 30$ & 13 & 6.2 & 3 & $5.4 \pm 1.1$ & $4.4 \pm 0.9$ & anti-phase \\
\hline
\end{tabular}

a Numbers after \pm represent spectral width $(0.5 \times$ FWHM $)$, see Sect. 2.

These intervals represent times when phasing between pearl wavepacket envelopes and Pc 5 wave cycles were visually observed to remain constant at approximately $0^{\circ}$-phase (inphase) or $180^{\circ}$-phase (anti-phase). The time intervals are summarised in Table 1 along with 12 August EMIC wave results. Section 3 includes discussion on the relationship between Pc 5 waves and pearl EMIC waves when the phase differences are not constant at $0^{\circ}$ or $180^{\circ}$.

Events 2 to 5, summarised in Table 1 are now described in more detail. During the 11 August event the trough of Pc 5 wave cycles corresponded to peaks in Pc 1 wavepacket envelopes before $\sim 05: 40$ UT, while after this time Pc 5 wave amplitudes are mostly in-phase with Pc 1 wavepacket envelopes (see Fig. 4c). There are two short intervals on 1 October (see Fig. 4f) which show clear constant in(anti)phase relationship between the Pc 5 wave cycle and Pc 1 wavepacket envelopes; the interval 18:40-18:55 UT is inphase, while the second interval from about 19:15-19:30 UT is anti-phase. The power spectra during the approximate time intervals of events 2 to 5 are shown in Fig. 5. The frequency coverage for all four time intervals displayed in Fig. 5 show strong correlation between Pc 5 wave frequencies and Pc 1 wavepacket envelope frequencies.

\subsection{Statistical results}

The EMIC wavepackets shown in Figs. 4 and 1 form part of the CRRES dataset previously studied by Loto'aniu et al. (2005). A visual search of the 248 EMIC wavepacket events presented in Loto'aniu et al. (2005) identified five EMIC pearl events from which 58 wavepackets were identified. However, Loto'aniu et al. (2005) were restricted in their 

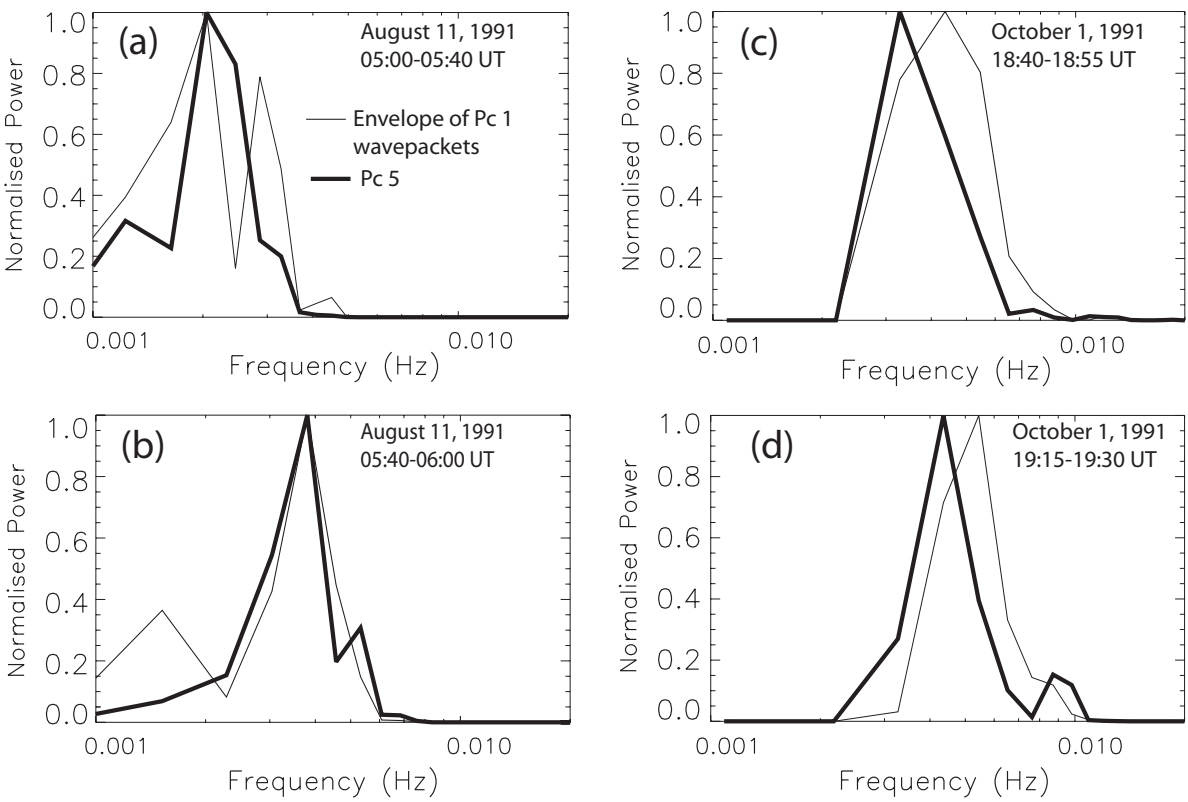

Fig. 5. Normalised power spectra for 11 August 1991 and 1 October 1991 time intervals. Thinlines represent spectra of Pc 1 transverse wavepacket envelopes, while thicklines are the spectra of Pc 5 waves.

event selection because of the requirement for both magnetic and electric field data for Poynting vector estimates. The events presented here should not be taken as an indicator of pearl occurrence in the middle magnetosphere.

Simultaneous Pc 5 waves were observed during all pearl events and the Pc 5 periods were measured over both Pc 1 wavepacket duration time intervals and repetition time intervals. Figure 6a shows the duration of Pc 1 wavepackets, $\tau_{\text {dur }}$, plotted against simultaneously occurring Pc 5 wave periods, $T_{\mathrm{Pc} 5}$. Similarly, Fig. 6b shows Pc 1 wavepacket repetition times versus Pc 5 periods observed over repetition intervals.

Simple linear regression analysis (lines through Fig. 6) gives a correlation coefficient of $R=0.7$ for Pc 1 wavepacket duration versus Pc 5 wave period. The best fit equation for Pc 1 wavepacket duration (in seconds) is

$\tau_{\mathrm{dur}}=0.8 \cdot T_{\mathrm{Pc} 5}+6$.

Hence, given that Pc 5 waves have a period range of 100 $500 \mathrm{~s}(2-10 \mathrm{mHz})$ pearl Pc 1 EMIC wavepacket durations should be about 1.4-6.8 $\min (86-406 \mathrm{~s})$. However, there was only a weak correlation between Pc 1 wavepacket repetition periods and Pc 5 wave period with $R=0.3$. Further discussion of Eq. (1) is presented in Sect. 3.

\section{Discussion}

In Sects. 2.1 and 2.2, pearl Pc 1 EMIC waves observed during three CRRES orbits were presented. The Poynting vector over each time interval was dominated by unidirectional propagation away from the equatorial region. However, some bidirectional energy propagation was observed on 1 October, close to the magnetic equator. As mentioned in Sect. 2, the Poynting vector results are consistent with Loto'aniu et al. (2005) who found bidirectional propagation to occur only within $\pm 11^{\circ} \mathrm{MLat}$, suggesting a wide generation region. Once outside this region EMIC wavepackets propagate unidirectionally away from the equator towards the ionosphere. These results cannot be explained by the BWP paradigm. Simultaneous ULF waves were observed during all pearl time intervals (see Figs. 1c and 4c and f).

The results in Sect. 2 suggest a relationship between the ULF waves and the observed pearls. However, currently little is known of how pearls can be generated through a process where ULF waves modulate parameters important to EMIC wave growth and propagation. In this section, discussion focuses on describing scenarios and mechanisms, given the results, where modulation may occur in order to highlight areas that need to be considered for future studies.

\subsection{Periodicity}

All five events summarised in Table 1 show Pc 5 wave frequencies $1 \mathrm{mHz}$ or less from the Pc 1 wavepacket envelope frequencies. Wave observations in the magnetosphere often show simultaneous presences of both Pc 1-2 waves and longer-period ULF pulsations (e.g. Fraser et al., 1992; Mursula et al., 1997; Rasinkangas and Mursula, 1998; Mursula et al., 2001). Recent studies suggest a relationship between periods of the ULF wave and repetition periods of the 
pearl EMIC waves (Rasinkangas and Mursula, 1998; Mursula, 2007). However, the spectra shown in Figs. 3 and 5 are the first direct spectral comparisons of Pc 1 wavepacket envelopes to simultaneously occurring Pc 5 waves. Results are generally consistent with the idea that Pc 5 ULF waves modulating parameters important to EMIC wave growth generate pearls with an envelope frequency close to the modulation frequency (e.g. Mursula et al., 1997).

The linear regression shown in Fig. 6a for 58 wavepackets suggest a linear relationship between pearl wavepacket duration $\left(\tau_{\mathrm{dur}}\right)$ and the Pc 5 wave periods $\left(T_{\mathrm{Pc} 5}\right)$. This correlation was low for EMIC wavepacket repetition times (Fig. 6b), which may suggest ULF waves do not influence initial generation of EMIC waves but rather sustainability of wavepacket growth. In other words, generation parameters (e.g., increased temperature anisotropy) provides necessary conditions for EMIC wave growth and ULF waves modulating these parameters influence how long wavepacket growth will last.

As mentioned in Sect. 2.3, given Pc 5 waves have periods of 100-500 s Eq. (1) suggests Pc 1 wavepacket durations should be $\sim 1.4-6.8 \mathrm{~min}$. However, Pc 3-4 waves have been found to correlate with pearl EMIC waves of durations less than about 2 min (e.g. Plyasova-Bakouniva et al., 1996; Mursula et al., 1997; Rasinkangas and Mursula, 1998; Mursula et al., 1999). Therefore, an obvious extension of this statistical study would be to fill in the lower left corner of Fig. 6a with observations of Pc 3-4 waves modulating pearls.

\subsection{Phase}

On 12 August, the pearl wavepacket envelope was observed to be constantly $180^{\circ}$ out of phase with the Pc 5 wave cycle. The other two observation days show constant phase difference of either $180^{\circ}$ or $0^{\circ}$ only during certain time intervals, which are summarised in Table 1 . The time intervals shown in the table range from $15-40 \mathrm{~min}$. This may suggest that inhomogeneous conditions in the magnetosphere probably only allow phase differences between EMIC wavepackets and Pc 5 ULF waves to remain constant for about $40 \mathrm{~min}$ or less.

Assuming $\mathrm{H}^{+}$ion plasma, the resonant kinetic energy of the interacting protons (Kennel and Petschek, 1966) can be written as

$E_{R}=\frac{B_{o}{ }^{2}}{2 m \cdot N_{e}} \frac{1}{X^{2}}(1-X)^{3}$,

where $N_{e}$ is electron number density, $m$ is the ion mass, $X$ is wave frequency normalised to the equatorial $\mathrm{H}^{+}$cyclotron frequency and $B_{o}$ is the background magnetic field. Intervals of anti-phase correlation may indicate modulation of EMIC wave linear growth rate by a decreasing $B_{o}$ due to the negative cycle of the ULF wave period, decreasing Alfvén speed, $V_{a}$, and resonant energy, $E_{R}$. A decrease in $E_{R}$ provides more energetic ions for cyclotron instability,
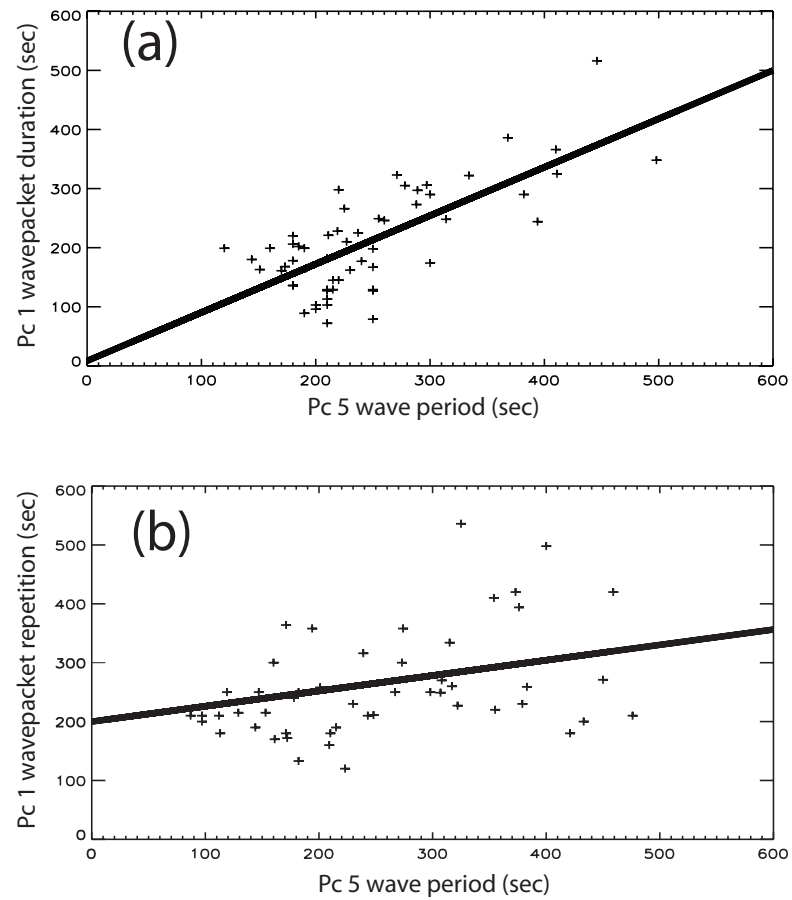

Fig. 6. The Pc 1 wavepacket duration (a) and repetition (b) periods versus Pc 5 wave periods. Note, the 58 wavepacket duration times were taken from 5 pearl events, which corresponds to 53 repetition intervals in (b). Also, since Pc 5 wave periods were measured over both duration and repetition time intervals, the Pc 5 wave period values shown in (a) do not necessarily equal those shown in (b).

while the wave spends more time in the generation region when $V_{a}$ decreases. The 12 August event occurred within an enhanced plasma density region resulting in a further decrease in Alfvén velocity.

Other critical parameters such as temperature anisotropy and cold plasma density may also be modulated by ULF waves (Rasinkangas et al., 1994). However, modulation of temperature anisotropy mostly supports in-phase modulation. When assuming adiabatic interaction between energetic ions and Pc 5 waves theoretical results favour no-phase difference between thermal anisotropy, $A$, and the magnetic field for EMIC wave generation (e.g. Gokhberg et al., 1981; Lyatsky and Plyasova-Bakunina, 1986). An early theoretical study by Coroniti and Kennel (1970) assuming an adiabatic process considered modulation of generation parameters of VLF whistlers by Pc 3-5 ULF waves using the relationship

$\left(\frac{\partial A}{\partial t}\right)_{\text {adiabatic }}=\frac{1+A}{B_{o}} \cdot b(t)$

where $b(t)$ is the time dependent field variation which is in-phase with variations in $A$. This adiabatic process may explain the two in-phase event intervals shown in Table 1 . In other words, Pc 5 wave modulation of temperature anisotropy may provide a mechanism for generating pearl 
EMIC waves that have wavepacket envelopes in-phase with the Pc 5 wave cycle.

Kivelson and Southwood (1985) conducted a theoretical study of the relationship between energetic ions and compressional Pc 5 waves. They found that although betatron heating provides ion flux oscillations in phase with variations in the magnetic field, some phase difference does occur between ion flux oscillations and the magnetic field in the resonant response. Hence, if one considers non-adiabatic processes EMIC waves may be modulated at varying phase differences with respect to the Pc 5 wave period. This may explain the varying phase differences observed during $11 \mathrm{Au}-$ gust and 1 October; both events occurred shortly after or during sudden storm commencement, which are favourable conditions for magnetospheric non-adiabatic processes.

In the treatment of Pc 5 ULF modulation of EMIC waves, nonlinear interactions may also be significant. Gail (1990) found that even small changes in the plasma parameters are enough to cause large variations in wave growth within marginally stable regions. Mursula et al. (1997) proposed that modulation due to ULF waves of plasma close to this marginally stable region produces the pearl structure. Gail studied effects of time dependent perturbation of magnetospheric plasma and field parameters on electromagnetic cyclotron waves. Assuming ideal MHD for long-period oscillations and using quasi-linear theory, Gail rewrote the refractive index as

$n^{2}=n_{o}^{2}(1+N(b))$

where $n_{o}$ is the index of refraction assuming cold plasma and $N(b)$ is the perturbed part of the refractive index due to time dependent variations in magnetic field. By redefining the temporal growth rate as

$\gamma \simeq \gamma_{o}(1+\Gamma)$

where $\Gamma$ is the modulation index and includes the effect of wave growth due to perturbation, $b$, Gail found that positive growth rates could occur for equilibrium anisotropy which are less than critical anisotropy. Further, Gail suggested that for fixed equilibrium growth rates, much larger fluctuations in growth rate occur for marginally stable distribution than for unstable distributions.

Nonlinear effects might explain why the Pc 1 wave envelope frequencies and Pc 5 wave frequencies do not always match. Given a marginally stable region, spectral power overlapping regions may be sufficient to provide EMIC wave growth. However, it should also be noted that Gail's definition of a varying local growth rate and plasma density do not take into account varying phases between pearl wavepacket envelopes and ULF waves.

The simultaneously occurring Pc 5 waves presented in Sect. 2 are only the compressional magnetic field component of the Pc 5 waves. The transverse field components may also play an important role in modulation. Rasinkangas et al.
(1994) suggested that EMIC wave modulation is produced by the radial electric field of the Pc 5 wave, which modulates equatorial cold plasma density by moving plasma from higher to lower $L$-value affecting EMIC wave growth rate. However, they did not present any long-period wave electric field data. Assuming a process that is adiabatic and in-phase, Pc 5 transverse magnetic field components may slosh plasma in-and-out of the generation region providing a modulation mechanism. Hence, future studies should take into account the transverse components.

\subsection{Heavy-ion effects}

Recently, Mursula (2007) noted that modulation of EMIC waves by longer-period ULF waves should be dependent on the heavy ion content. This dependency is partly due to possible heavy ion effects upon the modulating ULF waves, and partly because some modulated properties of the heavy ions (e.g. density) may cause related variations in EMIC wave growth rate (Mursula, 2007). On 12 August, EMIC waves were observed with wave frequency in the $\mathrm{He}^{+}$band, while wave frequencies observed on 11 August and 1 October occurred predominately in the $\mathrm{H}^{+}$band. The presences of $\mathrm{O}^{+}$ and $\mathrm{He}^{+}$ions should affect propagation of the $\mathrm{He}^{+}$and $\mathrm{H}^{+}$ band waves (e.g. Young et al., 1981; Rauch and Roux, 1982; Perraut et al., 1984).

On 12 August, EMIC waves were observed inside an enhanced plasma region (see Fig. 2) indicating plasmaspheric refilling due to an SSC on the previous day. During storm recovery $\mathrm{O}^{+} / \mathrm{H}^{+}$ratio usually increases (e.g. Horwitz et al., 1984). The Dst index (http://swdcwww.kugi.kyoto-u.ac.jp/) reached a minimum of -58 at 08:00 UT on 12 August, then retreated to -28 at 13:00 UT before reaching a second minimum of -99 at 20:00 and 22:00 UT on that day. Hence, it is reasonable to suggest that significant $\mathrm{O}^{+}$ions were present during generation of this $\mathrm{He}^{+}$band pearl. This event was also observed near critical off-equatorial bi-ion and cutoff frequency locations. Modulation near these critical frequency locations may produce propagation stop bands inbetween pearl wavepackets. However, assuming the background field is modulated by a $2 \mathrm{nT}$ ULF wave (see Fig. 1c) the local $\mathrm{O}^{+}$cyclotron frequency changes by less than $1 \%$. Furthermore, the power spectra and unidirectionally propagating Poynting vector results suggest critical off-equatorial heavy-ion frequencies had little effect on wave energy propagation. Hence, it is unlikely that propagation effects due to heavy-ions could have produced the clear pearl wavepacket structure seen on 12 August. A similar argument can be made regarding pearl EMIC waves observed well away from the equator on 11 August. It is more likely that the pearls were created close to the generation region possibly through modulation of the wave growth rate.

The $\mathrm{H}^{+}$band pearl EMIC waves on 1 October were observed inside the near-equatorial generation region. Previous studies show that for a $\left(\mathrm{H}^{+}, \mathrm{He}^{+}\right)$-plasma, enhancement in 
cold $\mathrm{He}^{+}$ion density leads to large increases in the local temporal growth rate of EMIC waves (e.g. Märk, 1974; Young, 1979; Gendrin and Roux, 1980). Field-line mass loading by heavy ions should decrease natural field-line resonance (FLR) frequencies and result in lower frequency ULF waves. This would produce a longer ULF wave period and according to Eq. (1) longer duration pearl wavepackets should be observed. In Sect. 3.2 it was noted that Pc 5 transverse magnetic field components may adiabatically move ions in-andout of the generation region. Ring current $(10-15 \mathrm{keV}) \mathrm{H}^{+}$ and $(100-300 \mathrm{keV}) \mathrm{O}^{+}$ions have been theoretically shown to generate ULF waves through a drift bounce mechanism (Ozeke and Mann, 2008). Hence, ULF wave interaction with energetic ions could modulate this ion population that provides the free energy for EMIC wave growth. Clearly, further studies using increase numbers of pearl events and theoretical simulations are required to understand the relationship between heavy-ion content and ULF wave modulation of EMIC waves.

\section{Conclusion}

This study has shown a clear frequency/periodicity relationship between pearl Pc 1 EMIC wave envelopes and simultaneously observed compressional Pc 5 ULF waves. The results also emphasis the need for detailed theoretical studies of the modulation of EMIC waves by ULF waves. This modulation process must take into account non-constant phase differences between pearl wavepackets and ULF waves. As a result, the process is likely to be highly complex including modulation with no-phase difference of temperature anisotropy and modulation with $180^{\circ}$-phase difference of the background magnetic field. Non-adiabatic interactions and nonlinear processes must also be included. Heavy-ion effects should also be considered since they affect both ULF waves and EMIC waves.

Acknowledgements. This work was partially carried out in the Cooperative Research Centre for Satellite Systems with financial support from the Commonwealth of Australia through the CRC program, and the University of Newcastle, Australia. Thanks are due to R. A. Anderson for providing the CRRES electron density data and H. J. Singer for providing the AFGL magnetometer data. This work was completed at the Space Weather Prediction Center and partially supported by the NOAA GOES-R program.

Topical Editor I. A. Daglis thanks M. Engebretson and $\mathrm{H}$. Laakso for their help in evaluating this paper.

\section{References}

Anderson, R. R., Gurnett, D. A., and Odem, D. L.: CRRES plasma wave experiment, J. Spacecraft and Rockets, 29, 570-573, 1992.

Barfield, J. N. and McPherron, R. L.: Investigation of interaction between Pc1 and 2 and Pc5 micropulsations at the synchronous orbit during magnetic storm, J. Geophys. Res., 77, 4707-4719, 1972.
Bossen, M., McPherron, R. L., and Russell, C. T.: A statistical study of Pc1 magnetic pulsations at synchronous orbit, J. Geophys. Res., 81, 6083-6091, 1976.

Coroniti, F. V. and Kennel, C. F.: Electron precipitation pulsations, J. Geophys. Res., 75, 1279-1289, 1970.

Fraser, B. J., Samson, J. C., McPherron, R. L., and Russell, C. T.: Ion cyclotron waves observed near the plasmapause, Adv. Space. Res., 6, 223-226, 1986.

Fraser, B. J., Samson, J. C., Hu, Y. D., McPherron, R. L., and Russell, C. T.: Electromagnetic ion cyclotron wave observed near the oxygen cyclotron frequency by ISEE-1 and 2, J. Geophys. Res., 97, 3063-3074, 1992.

Fraser, B. J., Singer, H. J., Hughes, W. J., Wygant, J. R., Anderson, R. R., and Hu, Y. D.: CRRES Poynting vector observations of electromagnetic ion cyclotron waves near the plasmapause, J. Geophys. Res., 101, 15331-15343, 1996.

Gail, W. B.: Theory of electromagnetic cyclotron wave growth in a time-varying magnetoplasma, J. Geophys. Res., 95, 1908919097, 1990.

Gendrin, R. and Roux, A.: Energization of helium ions by protoninduced hydromagnetic waves, J. Geophys. Res., 85, 4577-4586, 1980.

Gokhberg, M. B., Pilipenko, V. A., Pokhotelov, O. A., and Troitskaya, V. A.: On the problem of the interaction between Pc1/Pi1 and Pc4-5, hydromagnetic waves, J. Geophys. Res., 86, 833-836, 1981.

Gurnett, D. A. and Inan, U. S.: Plasma waves observations with Dynamic Explorer 1 spacecraft, Res. Geophys., 26, 285-316, 1988.

Horwitz, J. L., Comfort, R. H., and Chappell, C. R.: Thermal ion composition measurements of the formation of the new outer plasmasphere and double plasmapause during storm recovery phase, Geophys. Res. Lett., 11, 701-704, 1984.

Jacobs, J. A. and Watanabe, T.: Micropulsation whistlers, J. Atmospheric Terrest. Phys., 26, 825-829, 1964.

Jacobs, J. A., Kato, Y., Matsushita, S., and Troitskaya, V. A.: Classification of geomagnetic pulsations, J. Geophys. Res., 69, 180$181,1964$.

Kennel, C. F. and Petschek, H. E.: Limit of stability trapped particle fluxes, J. Geophys. Res., 71, 1-28, 1966.

Kivelson, M. G. and Southwood, D. J.: Charged particle behaviour in low-frequency geomagnetic pulsations, 4. Compressional waves, J. Geophys. Phys., 90, 1486-1498, 1985.

Loto'aniu, T. M., Fraser, B. J., and Waters, C. L.: The Propagation of electromagnetic ion cyclotron wave energy in the magnetosphere, J. Geophys. Res., 110, A07214, doi:10.1029/ 2004JA010816, 2005.

Lyatsky, V. B. and Plyasova-Bakunina, T. A.: Influence of the Pc4 magnetic pulsations on Pc1 generation, Geomagnetism and Aeronomy, 26, 674-677, 1986.

Maltzeva, N., Troitskaya, V. A., Schepetnov, R., Pokhotelov, O., Gokhberg, M., Pilipenko, V., McPherron, R. L., and Barfield, N. J.: Pc4-Pc1 magnetic pulsations at synchronous orbit and their relation to pulsations on the ground, J. Geophys. Res., 87, 10439-10448, 1982.

Märk, E.: Growth rates of the ion cyclotron instability in the magnetosphere, J. Geophys. Res., 79, 3218-3220, 1974.

Mauk, B. H. and McPherron, R. L.: An experimental test of the electromagnetic ion cyclotron instability within the Earth's magnetosphere, Phys. Fluid, 23, 2111-2127, 1980. 
McPherron, R. L., Russell, C. T., and Coleman Jr., P. J.: Fluctuating magnetic fields in the magnetosphere, II. ULF waves, Space Sci. Rev., 13, 411-454, 1972.

Mursula, K.: Satellite observations of Pc 1 pearl waves: The changing paradigm, in: Pc1 Pearl Waves: Discovery, Morphology and Physics, J. Atmos. Sol-Terr. Phys., 69, 1623-1634, 2007.

Mursula, K., Rasinkangas, R., Bösinger, T., Erlandson, R. E., and Lindqvist, P.-A.: Nonbouncing Pc 1 wave bursts, J. Geophys. Res., 102, 17611-17624, 1997.

Mursula, K., Bräysy, T., Rasinkangas, R., Tanskanen, P., and Mozer, F.: A modulated multiband Pc1 event observed by Polar/EFI around the plasmapause, Adv. Space Sci., 24, 81-84, 1999.

Mursula, K., Bräysy, T., Niskala, K., and Russell, C. T.: Pc1 pearl revisited: Structured electromagnetic ion cyclotron waves on Polar satellite and on ground, J. Geophys. Res., 106, 29543-29553, 2001.

Obayashi, T.: Hydromagnetic whistlers, J. Geophys. Res., 70, 1069-1078, 1965.

Ozeke, L. G. and Mann, I. R.: Energization of radiation belt electrons by ring current ion driven ULF waves, J. Geophys. Res., 113, A02201, doi:10.1029/2007JA012468, 2008.

Perraut, S., Gendrin, R., Roux, A., and de Villedary, C.: Ion cyclotron waves: Direct comparison between ground-based and observations in the source region, J. Geophys. Res., 89, 195-202, 1984.

Plyasova-Bakouniva, T. A., Kangas, J., Mursula, K., Molchanov, O. A., and Green, A. W.: Pc 1-2 and Pc 4-5 pulsations observed at a network of high-latitude stations, J. Geophys. Res., 101, 10965-10973, 1996.

Polyakov, S. V., Rapport, V. O., and Trakhtengerts, V. Y.: Alfvén sweep-maser, Sov. J. Plasma Phys., 9, 371-378, 1983.

Rasinkangas, R. and Mursula, K.: Modulation of magnetospheric EMIC waves by Pc3 pulsations of upstream origin, Geophys. Res. Lett., 25, 869-872, 1998.
Rasinkangas, R., Mursula, K., Kremser, G., Singer, H. J., Fraser, B. J., Korth, A., and Hughes, W. J.: Simultaneous occurrence of Pc5 and Pc1 pulsations in the dawnside magnetosphere: CRRES observations, in: Solar wind sources of magnetopsheric UltraLow-Frequency waves, Geophysical Monograph 81, 417-424, 1994.

Rauch, J. L. and Roux, A.: Ray tracing of ULF waves in a multicomponent magnetospheric plasma: Consequences for the generation of ion cyclotron waves, J. Geophys. Res., 87, 8191-8198, 1982.

Saito, T.: Geomagnetic pulsations, Space Science Rev., 10, 319412, 1969.

Singer, H. J., Sullivan, W. P., Anderson, P., Mozer, F., Harvey, P., Wygant, J., and McNeil, W.: Fluxgate magnetometer instrument on the CRRES, J. Spacecraft and Rockets, 29, 599-601, 1992.

Tepley, L.: Low-latitude observations of fine-structured hydromagnetic emissions, Geophys. Res., 69, 2273-2290, 1964.

Tepley, L. and Landshoff, R. K.: Waveguide theory for ionospheric propagation of hydromagnetic emissions, J. Geophys. Res., 71, 1499-1504, 1966.

Troitskaya, V. A.: Pulsations of the Earth's electromagnetic field with periods of 1 to 15 seconds and their connection with phenomena in the high atmosphere, J. Geophys. Res., 66, 5-18, 1961.

Wygant, J., Harvey, P. R., Pankow, D., Mozer, F. S., Maynard, N., Singer, H., Smiddy, M., Sullivan, W., and Anderson, P.: The CRRES electric field/Langmuir probe instrument, J. Spacecraft and Rockets, 29, 601-604, 1992.

Young, D. T.: Ion composition measurements in magnetospheric modelling, in: Quantitative modelling of magnetospheroc processes, edited by: Olson, W. P., p. 340, American Geophysical Union, American Geophysical Union, Washington D.C., 1979.

Young, D. T., Perraut, S., Roux, A., de Villedary, C., Gendrin, R., Korth, A., Kremser, G., and Jones, D.: Wave-particle interactions near $\Omega_{\mathrm{He}}+$ observed on GEOS 1 and 2, 1. Propagation of ion cyclotron waves in $\mathrm{He}^{+}$-rich plasma, J. Geophys. Res., 86, 6755-6772, 1981. 